\title{
Identifying predictors to gain sensitivity of nucleic acid amplification tests for Mycobacterium tuberculosis
}

\section{Xingfang Hou}

First Hospital of Changsha

\section{Qinglong Guo}

The Third People's Hospital of Shenzhen

Qiao Lin

Southern Medical University

\section{Taosheng Ye}

The Third People's Hospital of Shenzhen

\section{Jing Bi}

The Third People's Hospital of Shenzhen

\section{Jing Bi}

The Third People's Hospital of Shenzhen

\section{Juan Liang}

Jinan University

\section{Ke Yang}

The First Hospital of Changsha

\section{Yuan Zhou}

The First Hospital of Changsha

\section{Juanjuan Zhang}

The Third People's Hospital of Shenzhen

\section{Zhihang Liang}

The Third People's Hospital of Shenzhen

\section{Xuefeng Zhou}

The Third People's Hospital of Shenzhen

\section{Gengwei Zhang}

The Third People's Hospital of Shenzhen

\section{Xiangdong Fu}

The Third People's Hospital of Shenzhen

\section{Hongjian Zhong}

Southern Medical University

Guoliang Zhang ( $\nabla$ szdsyy@aliyun.com ) 
national clinical research center for infectious dieseases, shenzhen third people's hospital, southern university of science and technology, shenzhen, china

\section{Research Article}

Keywords: pulmonary tuberculosis, nucleic acid amplification tests, indicators, detection, Mycobacterium tuberculosis

Posted Date: November 18th, 2020

DOl: https://doi.org/10.21203/rs.3.rs-105455/v1

License: (c) (i) This work is licensed under a Creative Commons Attribution 4.0 International License. Read Full License 


\section{Abstract}

Background: Tuberculosis (TB) remains one of the primary threatening of human health and its diagnosis remains unsatisfactory in clinic. Nucleic acid amplification tests (NAAT) showed higher sensitivity than culture for the diagnosis of pulmonary TB (PTB). However, NAAT are expensive and not easily deployable at the peripheral level. To improve the sensitivity of NAAT for the PTB diagnosis, the predictive factors that might be utilized to give the optimized choice of NAAT were investigated.

Methods: A total of 1263 PTB suspects were enrolled for evaluation. The sensitivity, specificity and accuracy of Mtb detection in sputum and bronchoalveolar lavage fluid (BALF) were compared. Odds ratios and $95 \%$ confidence intervals were used to assess variables that associated with positive NAAT in sputum and BALF of PTB suspects.

Results: An significantly enhanced sensitivity was observed when performed on NAAT (61.1\%) compared with smear (9.0\%) and culture of Mtb (47.8\%). We found that erythrocyte sedimentation rate (ESR) (+), cavities $(+)$ and IFN- $y$ release assay (IGRA) $(+)$ are involved in the positivity of Mtb detection through NAAT. Moreover, those who are ESR (+), cavities $(+)$ and IGRA $(+)$, showed $86 \%$ diagnostic positivity of Mtb by NAAT.

Conclusions: Our study suggested that combination of the results of ESR and IGRA and the presence of pulmonary cavity is helpful to predict the positivity of Mtb detection through NAAT. Those who are ESR $(+)$, cavities (+) and IGRA (+), should perform NAAT for Mtb detection, because they are most likely to be bacteriologically confirmed as TB.

\section{Background}

TB, caused by Mycobacterium tuberculosis (Mtb), remains one of the primary threatening of human health in the world. There were approximate 10 million new TB cases and caused 1.45 million TB deaths in 2018(1). Given that Bacille Calmette-Guerin (BCG), the only licensed vaccine, has variable efficacy that gives protection against childhood TB but is not valid in adult pulmonary TB (PTB)(2), the timely and rapid diagnosis of PTB is crucial for individual anti-TB treatment as well as could efficiently prevent the transmission of TB.

Culture of the bacteria is the gold standard for TB diagnosis, but it has low sensitivity, high contamination rate and needs long growth period and thus results in the delay of anti-TB treatment and increases the exposure of the TB patients to the healthy population(3). Acid-fast bacilli smear is fast and cheap, while has low sensitivity and specificity $(4,5)$. Mtb-antigen specific IGRA has higher sensitivity, but it can not discriminate active TB (ATB) from dormant TB infections $(6,7)$. Thus, it is eager to identify optimal methods for PTB diagnosis.

As a newer method, NAAT that has been developed during the last decades, is a promising diagnostic technique of PTB that shows higher sensitivity and specificity. For example, the widely used and WHO- 
endorsed Xpert@MTB/RIF assay (Cepheid, Sunnyvale, CA, USA) is an automated, single cartridge-based NAAT and can rapidly detect TB and rifampicin resistance $(8,9)$. Although providing the possibility to increase the positive rate and save time for the diagnosis of PTB, NAAT is costly and not easily deployable at the peripheral level. Thus, ascertaining factors to speculate the yield of NAAT for PTB diagnosis is necessary to obtain the maximized benefit from NAAT but minimizing the expense to TB control programs. Here, the sensitivity and specificity between culture and NAAT for PTB diagnosis were compared and clinical factors involved in the benefit of NAAT in discriminating PTB were also confirmed.

\section{Methods And Materials}

\section{Study population}

This study that included a total of 1263 PTB suspects collected from February 2011 to June 2015 was retrospectively performed at Shenzhen Third People's Hospital (Guangdong, China). latrical data were collated including age, sex, premonitors, previous TB history, lab tests and imaging examination. Lab experiments used in the present study contained sputum smear, NAAT, culture, IGRA, and CD4 and CD8 cell calculation. Patients with a follow-up lacking 6 months were precluded. The study was agreed by the Institutional Review Board of Shenzhen Third People's Hospital. Written informed consent were gained from every patients.

\section{Diagnosis of PTB}

The clinical premonitor, radiological sign indicative of PTB, culture of Mtb, microscopy, NAAT, and response to anti-TB therapy were used for the diagnosis of PTB. Both sputum and BALF collected from every patients were used to detect Mtb by microscopy, culture of Mtb and NAAT. Patients who were sputum or BALF culture $(+)$ for Mtb, or NAAT $(+)$ for Mtb, plus clinical premonitor and radiological sign indicative of PTB, were confirmed as definite TB. Patients who were culture (-), NAAT (-) and AFB (-) for Mtb both in sputum and BALF, plus clinical premonitor, radiological sign indicative of PTB and response to anti-TB therapy, were confirmed as probable TB. The PTB patients who had finished standard anti-TB therapy for no less than 6 months without subsequent proof of ATB were confirmed as cured TB. Non-TB lung disease (non-TB) was demonstrated according to having no microbiological or no histological proof of PTB or demonstration of an alternative diagnosis or a lack of progression to PTB more than 6 months of followed-up without PTB therapy(10). Non-tuberculosis mycobacteria (NTM) infection was defined according to the confirmation of NTM from the same species in sputum and/or BALF.

\section{Specimen acquisition, processing and detection}

To collect BALF, isotonic saline was used for bronchial washing of the related sub-segment. All sputum and BALF samples were performed on microscopy, NAAT and culture of Mtb in Lowenstein-Jensen medium and BACTEC $9000 \mathrm{MB}$ liquid medium for the confirmation of Mtb. The IGRA was determined as reported previously using an in-house IFN-y enzyme-linked immunoblot assay, which the sensitivities and specificities was similar to that of commercial available kits(6). 


\section{Statistical analysis}

For identification of potential variables involved in positivity of NAAT (positivity in Mtb examination in sputum and BALF through NAAT), single variable analysis with Pearson's $\chi^{2}$ test was carried out. Every variable with $P<0.05$ in single variable analysis were subjected to the unconditional multiple logistic regression analysis and the associations were evaluated by odd ratios $(O R)$ and $95 \%$ confidence intervals $(\mathrm{Cls})$ and those variables with $P<0.05$ were subjected to stratified analysis for identification of the superimposed roles on positivity of NAAT. All analysis were conducted by SPSS 26.0 .

\section{Results}

\section{Study population}

A total of 1263 patients were enrolled in this study. 1173/1263 patients were diagnosed with PTB, 18/1263 patients were cured TB without TB relapse, 16/1263 cases were infected with NTM, and $56 / 1263$ patients were discriminated with non-TB lung diseases (Table 1). The average age was 35.96 and $57.4 \%$ were males.

\section{NAAT enhanced the diagnostic accuracy of PTB suspects}

As shown in Table 2, sensitivity and specificity etc. of NAAT, culture and smear for the diagnosis of PTB patients were determined. Among the 1263 patients, NAAT showed significant higher positive detectable rate for the diagnosis of PTB suspects in comparison to that of smear and culture of Mtb, with the positivity of $58.0 \%, 8.5 \%$ and $44.4 \%$, respectively (Table 2 ). An significantly enhanced sensitivity was observed when performed on NAAT (61.1\%) compared with smear (9.0\%) and culture of Mtb $(47.8 \%)$ (Table 2). Similarly, the accuracy of NAAT $(62.6 \%)$ was significantly higher than that of smear $(15.4 \%)$ and culture of Mtb (51.5\%) (Table 2, Table S1). Taken together, these results suggested that the method of NAAT could better contribute to the diagnosis of PTB suspects.

\section{Factors involved in positive Mtb detection through NAAT in PTB}

Consistent with previous reports(11), a significantly increased Mtb detection rate in PTB suspects assayed on NAAT was observed. 717/1173 (61.1\%) patients were positive for Mtb detection performed on NAAT. Although greatly contributed to the PTB diagnosis and drug susceptibility testing, NAAT test is costly and not easily deployable at the peripheral level. For identification of the patients most probably to profit from NAAT test, factors involved in Mtb examination of PTB through NAAT were analyzed. We found that lose weight $(P=0.020)$, bilateral lung infection $(P=0.018)$, cavity $(P<0.001)$, ESR $(P<0.001)$, IGRA results $(P<0.001)$ were all markedly involved in Mtb examination of PTB through NAAT test when performed univariate analysis (Table 3 ). Furthermore, our results showed that cavity (OR: $2.317,95 \% \mathrm{Cl}$ : 1.640-3.275), IGRA results (OR: 2.651, 95\% Cl: 1.861-3.776) and ESR (OR: 3.088, 95\% Cl: 2.134-4.469) were independent risk factors related to the positive detection of Mtb of PTB through NAAT test when performed multivariate logistic regression analysis (Table 4). Further analysis suggested that the 
combined results of ESR, cavity and IGRA gained the highest positivity of Mtb examination of PTB by NAAT test (Table 5).

\section{Discussion}

The early diagnosis and timely treatment are necessary for efficient TB control. The failure to rapidly recognize and cure affected patients caused improved mortality, secondary resistance, and sustaining transmission(11). The faster NAAT test has brought about a shorter time for TB diagnosis and, thus, rapider anti-TB treatments. In the present study, the sensitivity of NAAT test was $61.1 \%(717 / 1173)$ in sputum and BALF of PTB patients, while the sensitivity of smear and culture was $9.0 \%(106 / 1173)$ and $47.8 \%$ (561/1173) respectively. Besides, 16 NTM infected patients were determined through NAAT tests. Together, about $50 \%$ and $15 \%$ patients benefited from NAAT tests compared with smear and culture, respectively. NAAT test improved the positive detectable rate of PTB diagnosis as well as contributed to the effective anti-TB therapy by identifying species and drug susceptibility testing(12).

Although fast, safe, convenient and accurate, NAAT test are expensive and not easily deployable at the peripheral level, such as MTB/RIF test(13). The MTB/RIF test employs sophisticated technology to obtain great simplicity of use, which is expensive to manufacture. Moreover, the need for annual calibration was difficult to implement at peripheral laboratories, particularly in rural areas. Thus, we identified factors correlated with the positive detection of Mtb of PTB through NAAT test trying to maximize the profit of PTB patients who performed NAAT test. We found that risk factors related to positivity of Mtb detection through NAAT tests were ESR, cavity and IGRA results. Those factors play an overlapping role in predicting positivity of Mtb examination through NAAT test with the highest positivity of $86 \%$ (a combination of ESR (+), cavity (+) and IGRA (+)) for Mtb detection.

ESR gets the highest predictive value among them. The ESR test is widely used as nonspecific test for many pathological conditions such as infections and inflammatory diseases. Infections could elevate the value of ESR and are said to be one of the primary reasons of elevated values $\geq 100 \mathrm{~mm} / \mathrm{h}(14-16)$. A previous study suggested that ATB was mainly involved in extremely high ESR values $(\geq 100 \mathrm{~mm} / \mathrm{h})(16)$. Another study also found that TB patients without HIV infection had the mean and median values more than $100 \mathrm{~mm} / \mathrm{h}$ (17). In line with these studies, our results suggested that the elevated values of ESR were associated with ATB and therefore, associated with Mtb detection through NAAT test. Mtb infections could often result in the formation of cavities in the lungs of patients and the bacilli grows on the cavity wall and enters into the bronchial tree, thus enabling them spread to other parts of the lung and also infect other people(18). Positive IGRA results was also predictive factors for Mtb detection through NAAT test. Our recent result found that the degree of IFN- $\gamma$ response in BALF of PTB determined by IGRAs was positively associated with bacteria load(19). Moreover, other researchers reported that the degree of IFN- $\gamma$ are involved in sputum positivity in immune competent PTB patients $(12,20)$. Consistent with these results, our result showed that IGRA results gives a fatidic odds ratio of 2.651 (95\% Cl: 1.861-3.776) for positivity of Mtb detection through NAAT test. In summary, we predicted that patients with higher ESR are 
more likely ATB enabling to mount a robust Mtb-specific IFN-y response and develop cavities with positive detection in both sputum and BALF for Mtb through NAAT test.

\section{Conclusions}

Our study suggested that combination of the results of ESR and IGRA and the presence of pulmonary cavity is helpful to predict the positivity of Mtb detection through NAAT. Those who are ESR (+), cavities $(+)$ and IGRA (+), should perform NAAT for Mtb detection, because they are most likely to be bacteriologically confirmed as TB.

\section{Declarations}

\section{Funding source}

This work was supported by the Natural Science Foundation of China (No. 81873958, 81802058); National Key Research and Development Plan (No. 2019YFC0840602); the Guangdong Fundamental and Applied Fundamental Research Project (No. 2019B1515120041); the Shenzhen Scientific and Technological Foundation (No. JCYJ20180228162336873, JCYJ20180228162321234); China Postdoctoral Science Foundation (No. 2020M670085ZX).

\section{Conflict of interest}

None

\section{Author's contributions}

X-FH, Q-LG, QL, JB, JL, J-JZ, Z-HL, X-FZ and G-WZ performed the study. X-FH, Q-LG and QL analyzed the data and wrote the manuscript. T-SY, KY and YZ provided clinical data. X-DF, H-JZ and G-LZ supervised the study and applied for grants. X-FH, Q-LG, QL and G-LZ were identified as the guarantor of the paper, taking responsibility for the integrity of the work as a whole, from inception to published article. All authors read and approved the final manuscript.

\section{Availability of data materials}

The dataset used and/or analyzed during the current study are available from the corresponding author on reasonable request.

\section{Ethics approval and consent to participate}

The study was approved by the Institutional Review Board of Shenzhen Third People's Hospital, and informed consent was obtained from each participant.

\section{Consent for publication}


Not applicable.

\section{Acknowledgements}

We would like to thank all study participants who were involved and contributed to the data collection. We thank editors and reviewers for their warm works and valuable comments.

\section{References}

1. Organization WH. Global tuberculosis report 2018. Geneva, Switzerland: World Health Organization; 2018. WHO/CDS/TB/2018.20. Available from: http://apps. who. int/iris/bitstream ...; 2018.

2. Dockrell HM, Smith SG. What Have We Learnt about BCG Vaccination in the Last 20 Years? Frontiers in immunology. 2017;8:1134.

3. Hanna BA, Ebrahimzadeh A, Elliott LB, Morgan MA, Novak SM, Rusch-Gerdes S, et al. Multicenter evaluation of the BACTEC MGIT 960 system for recovery of mycobacteria. Journal of clinical microbiology. 1999;37(3):748-52.

4. Newton SM, Brent AJ, Anderson S, Whittaker E, Kampmann B. Paediatric tuberculosis. The Lancet Infectious diseases. 2008;8(8):498-510.

5. Daniel TM. The rapid diagnosis of tuberculosis: a selective review. The Journal of laboratory and clinical medicine. 1990;116(3):277-82.

6. Chen X, Yang Q, Zhang M, Graner M, Zhu X, Larmonier N, et al. Diagnosis of active tuberculosis in China using an in-house gamma interferon enzyme-linked immunospot assay. Clinical and vaccine immunology : CVI. 2009;16(6):879-84.

7. Metcalfe JZ, Everett CK, Steingart KR, Cattamanchi A, Huang L, Hopewell PC, et al. Interferon-Y release assays for active pulmonary tuberculosis diagnosis in adults in low- and middle-income countries: systematic review and meta-analysis. The Journal of infectious diseases. 2011;204 Suppl 4(Suppl 4):S1120-9.

8. Trébucq A, Devi S. Widespread use of Xpert(®) MTB/RIF will most probably reduce the total number of TB cases notified. The international journal of tuberculosis and lung disease : the official journal of the International Union against Tuberculosis and Lung Disease. 2015;19(11):1406.

9. Greco S, Girardi E, Navarra A, Saltini C. Current evidence on diagnostic accuracy of commercially based nucleic acid amplification tests for the diagnosis of pulmonary tuberculosis. Thorax. 2006;61(9):783-90.

10. Liao M, Yang Q, Zhang J, Zhang M, Deng Q, Liu H, et al. Gamma interferon immunospot assay of pleural effusion mononuclear cells for diagnosis of tuberculous pleurisy. Clinical and vaccine immunology : CVI. 2014;21(3):347-53.

11. Boehme CC, Nabeta P, Hillemann D, Nicol MP, Shenai S, Krapp F, et al. Rapid molecular detection of tuberculosis and rifampin resistance. The New England journal of medicine. 2010;363(11):1005-15. 
12. Liu X, Hou XF, Gao L, Deng GF, Zhang MX, Deng QY, et al. Indicators for prediction of Mycobacterium tuberculosis positivity detected with bronchoalveolar lavage fluid. Infectious diseases of poverty. 2018;7(1):22.

13. Pai M. Innovations in Tuberculosis Diagnostics: Progress and Translational Challenges. EBioMedicine. 2015;2(3):182-3.

14. Brigden ML. Clinical utility of the erythrocyte sedimentation rate. American family physician. 1999;60(5):1443-50.

15. Levay PF, Viljoen M. Erythrocyte sedimentation rates equal to or above $100 \mathrm{~mm} / \mathrm{h}$ in the pre-AIDS era. South African medical journal = Suid-Afrikaanse tydskrif vir geneeskunde. 2002;92(4):286-7.

16. Ukpe IS, Southern L. Erythrocyte sedimentation rate values in active tuberculosis with and without HIV co-infection. South African medical journal = Suid-Afrikaanse tydskrif vir geneeskunde. 2006;96(5):427-8.

17. Sarkar K, Baraily S, Dasgupta S, Bhattacharya SK. Erythrocyte sedimentation rate may be an indicator for screening of tuberculosis patients for underlying HIV infection, particularly in resourcepoor settings: an experience from India. Journal of health, population, and nutrition. 2004;22(2):2201.

18. Abe Y, Nakamura M, Oshika Y, Hatanaka H, Tokunaga T, Ohkubo Y, et al. Serum levels of vascular endothelial growth factor and cavity formation in active pulmonary tuberculosis. Respiration; international review of thoracic diseases. 2001;68(5):496-500.

19. Guo Q, Zhang J, Li G, Liu S, Xiao G, Bi J, et al. Elevated antigen-specific IFN-y responses in bronchoalveolar lavage fluid impervious to clinical comorbidities improve the pulmonary tuberculosis diagnosis. Tuberculosis (Edinburgh, Scotland). 2020;122:101942.

20. De Keyser E, De Keyser F, De Baets F. Tuberculin skin test versus interferon-gamma release assays for the diagnosis of tuberculosis infection. Acta clinica Belgica. 2014;69(5):358-66.

\section{Tables}

Table 1. The clinical characteristics of pulmonary TB suspects 


\begin{tabular}{|c|c|c|c|}
\hline Groups/subgroups & No. & Males/females & Mean age $\pm S D(y r)$ \\
\hline Active Pulmonary TB & 1173 & $668 / 505$ & $35.09 \pm 13.344$ \\
\hline Bacterial negative TB & 385 & $230 / 155$ & $37.18 \pm 13.288$ \\
\hline Bacterial positive TB & 788 & $438 / 350$ & $34.07 \pm 13.259$ \\
\hline Cured TB & 18 & $9 / 9$ & $43.22 \pm 9.143$ \\
\hline NTM & 16 & $5 / 11$ & $46.62 \pm 14.268$ \\
\hline Non-TB lung disease & 56 & $43 / 13$ & $48.73 \pm 14.880$ \\
\hline Pneumonia & 45 & $36 / 9$ & $49.44 \pm 14.022$ \\
\hline Others $^{a}$ & 11 & $7 / 4$ & $45.82 \pm 18.465$ \\
\hline Total & 1263 & $725 / 538$ & $35.96 \pm 13.736$ \\
\hline
\end{tabular}

TB, tuberculosis; NTM, non-tuberculosis mycobacteria.

a Others included carcinoma of the lungs, asthma, bronchiectasis, chronic obstructive pulmonary disease, etc.

Table 2. Comparison of sensitivity, specificity, etc. among smear, culture and NAAT for the diagnosis of pulmonary TB suspects.

\begin{tabular}{|lllll|}
\hline & Positive detectable rate (\%) & Sensitivity (\%) & Specificity (\%) & Accuracy (\%) \\
\hline Smear & 8.5 & 9.0 & 97.8 & 15.4 \\
Culture & 44.4 & 47.8 & 100.0 & 51.5 \\
NAAT & 58.0 & 61.1 & 82.2 & 62.6 \\
\hline Culture or NAAT & 63.7 & 67.2 & 82.2 & 68.2 \\
\hline
\end{tabular}

TB, tuberculosis; NAAT, nucleic acid amplification test.

Table 3. Comparison of clinical characteristics between NAAT positive and negative patients within pulmonary TB patients. 


\begin{tabular}{|c|c|c|c|c|}
\hline & $\begin{array}{l}\text { All patients } \\
\qquad \begin{array}{l}N=1173\end{array}\end{array}$ & $\begin{array}{c}\text { NAAT (+) } \\
n=717\end{array}$ & $\begin{array}{l}\text { NAAT }(-) \\
n=456\end{array}$ & $P$-value \\
\hline Male & 668 & 394 (59.0\%) & $274(41.0 \%)$ & 0.083 \\
\hline Fever & 362 & $225(62.2 \%)$ & $137(37.8 \%)$ & 0.833 \\
\hline Night sweat & 124 & 74 (59.7\%) & $50(40.3 \%)$ & 0.621 \\
\hline Lose weight & 294 & $198(67.3 \%)$ & 96 (32.7\%) & 0.020 \\
\hline Hemoptysis & 183 & $116(63.4 \%)$ & 67 (36.6\%) & 0.610 \\
\hline Bilateral lung & 568 & $367(64.6 \%)$ & 201 (35.4\%) & 0.018 \\
\hline Cavity ${ }^{a}$ & 300 & $223(74.3 \%)$ & 77 (25.7\%) & $<0.001$ \\
\hline$C D 4 \leq 500^{b}$ & 366 & $236(64.5 \%)$ & $130(35.5 \%)$ & 0.119 \\
\hline$C D 8 \leq 500^{b}$ & 601 & $377(62.7 \%)$ & 224 (37.3\%) & 0.245 \\
\hline $\operatorname{ESR}(+)$ & 687 & $482(70.2 \%)$ & $205(29.8 \%)$ & $<0.001$ \\
\hline $\operatorname{IGRA}(+)$ & 845 & $553(65.4 \%)$ & $292(34.6 \%)$ & $<0.001$ \\
\hline
\end{tabular}

NAAT, nucleic acid amplification test; ESR, erythrocyte sedimentation rate; IGRA, interferon- $\gamma$ release assays.

a Total $N=957$, NAAT $(+) n=574$, NAAT $(-) n=383$

${ }^{b}$ Total $N=829$, NAAT $(+) n=510$, NAAT (-) $n=319$

Table 4. Factors correlated with NAAT positivity in pulmonary TB patients were subjected to univariate and multivariate analysis. 


\begin{tabular}{|llllll|}
\hline & Total & NAAT (+) & NAAT (-) & P-value & OR (95\% CI) ${ }^{\text {a }}$ \\
\hline Cavity & & & & & \\
\hline No & 657 & $351(53.4 \%)$ & $306(46.6 \%)$ & 0.001 & \\
\hline Yes & 300 & $223(74.3 \%)$ & $77(25.7 \%)$ & & $2.317(1.640-3.275)$ \\
\hline IGRA & & & & $<0.001$ & \\
\hline Negative & 328 & $164(50.0 \%)$ & $164(50.0 \%)$ & & Reference \\
\hline Positive & 845 & $553(65.4 \%)$ & $292(35.6 \%)$ & & $2.651(1.861-3.776)$ \\
\hline ESR & & & & $<0.001$ & \\
\hline Negative & 312 & $140(44.9 \%)$ & $172(55.1 \%)$ & & Reference \\
\hline Positive & 861 & $577(67.0 \%)$ & $284(33.0 \%)$ & & $3.088(2.134-4.469)$ \\
\hline
\end{tabular}

NAAT, nucleic acid amplification test; ESR, erythrocyte sedimentation rate; IGRA, interferon-y release assays.

a Controlling for variables with $P<0.05$ in univariate analysis

Table 5. Stratified analysis of factors correlated with NAAT positivity in pulmonary TB patients. 


\begin{tabular}{|c|c|c|c|c|}
\hline & $\begin{array}{l}\text { NAAT (+) } n / N \\
(\%)\end{array}$ & $\begin{array}{l}\text { NAAT }(-) n / N \\
(\%)\end{array}$ & $\begin{array}{l}P \\
\text { value }\end{array}$ & $O R(95 \% C l)$ \\
\hline \multicolumn{5}{|l|}{ ESR/Cavity/IGRA } \\
\hline ESR (-)/Cavity (-)/IGRA (-) & 9/37 (24.3\%) & 28/37 (75.7\%) & $<0.001$ & Reference \\
\hline$(+)$ ESR (-)/Cavity (-)/IGRA & $45 / 122(36.9 \%)$ & $77 / 122(63.1 \%)$ & 0.161 & $1.818(0.788-4.196)$ \\
\hline${ }_{(-)}$ESR (-)/Cavity (+)/IGRA & $4 / 9(44.4 \%)$ & $5 / 9(55.6 \%)$ & 0.238 & $2.489(0.548-11.313)$ \\
\hline$\underset{(+)}{\operatorname{ESR}(-) / \text { Cavity }(+) / \text { IGRA }}$ & 18/35 (51.4\%) & 17/35 (48.6\%) & 0.020 & $3.294(1.210-8.970)$ \\
\hline$(-)$ ESR (+)/Cavity (-)/IGRA & 43/92 (46.7\%) & 49/92 (53.3\%) & 0.021 & $2.730(1.161-6.422)$ \\
\hline$(+)$ ESR (+)/Cavity (-)/IGRA & $\begin{array}{l}216 / 334 \\
(64.7 \%)\end{array}$ & $\begin{array}{l}118 / 334 \\
(35.3 \%)\end{array}$ & $<.001$ & $5.695(2.601-12.471)$ \\
\hline$(-)$ ESR (+)/Cavity (+)/IGRA & $32 / 57(56.1 \%)$ & $25 / 57(43.9 \%)$ & 0.003 & $3.982(1.595-9.945)$ \\
\hline$(+)$ ESR (+)/Cavity $(+) /$ IGRA & $\begin{array}{l}154 / 179 \\
(86.0 \%)\end{array}$ & $25 / 179(14.0 \%)$ & $<.001$ & $\begin{array}{l}19.164(8.095- \\
45.368)\end{array}$ \\
\hline
\end{tabular}

NAAT, nucleic acid amplification test; TB, tuberculosis; ESR, erythrocyte sedimentation rate; IGRA, interferon- $y$ release assays.

\section{Supplementary Files}

This is a list of supplementary files associated with this preprint. Click to download.

- TableS1.docx

- TableS1.docx 